\title{
Effect of hydrostatic pressure on degradation of $\mathrm{CdTe} / \mathrm{CdMgTe}$
}

\section{heterostructures grown by molecular beam epitaxy on GaAs substrate}

D. Wasik, M. Baj, J. Siwiec-Matuszyk, J. Gronkowski

Institute of Experimental Physics, Warsaw University, Hoża 69, 00-681 Warsaw, Poland

J. Jasiński

Materials Science Division, Lawrence Berkeley National Laboratory, 1 Cyclotron Rd, Berkeley, California 94720

G. Karczewski

Institute of Physics, Polish Academy of Sciences, Al. Lotników 32/46, 02-668 Warsaw, Poland

Abstract

We have shown that external hydrostatic pressure leads to the creation of structural defects, mainly in the vicinity of the II-VI/GaAs interface in the $\mathrm{CdTe} / \mathrm{Cd}_{1-\mathrm{x}} \mathrm{Mg}_{\mathrm{x}} \mathrm{Te}$ heterostructures grown by the molecular beam epitaxy method on GaAs substrates. These defects propagating across the epilayer cause permanent damage to the samples from the point of view of their electrical properties. In contrast, photoluminescence spectra are only weakly influenced by pressure. Our results shed light on the degradation process observed even without pressure in II-VI - based heterostructures. 


\section{INTRODUCTION}

Heterostructures based on II-VI semiconducting materials have attracted much attention in recent years due to their possible applications in optoelectronic devices such as lasers, light emitting diodes, etc. The potential hybridization of II-VI with III-V optoelectronics has led to significant efforts in optimizing the epitaxial growth of II-VI layers on GaAs substrates. One of the most important problems, which so far has not been fully resolved, concerns the degradation of II-VI semiconducting heterostructures over time. Studies of the mechanisms are of scientific interest and could help to understand and thus eliminate degradation mechanisms in II-VI devices. Several reports on ZnSe-based heterostructures have shown that formation of structural defects at the $\mathrm{ZnSe} / \mathrm{GaAs}$ interface plays a crucial role in the degradation process [1-7]. Strains induced by the lattice mismatch between the substrate and the epilayer lead to creation of many crystal defects (point defects, dislocations, V-shape stacking faults etc) near the interface. Initially, the degradation process starts by formation of new dislocations and other structural defects from near pre-existing ones (mainly stacking faults) which propagate along the epilayer.

In the case of low-dimensional structures based on CdTe, real progress in the material quality has been recently achieved. This improvement makes it possible, for example, to manufacture $\mathrm{CdTe} / \mathrm{Cd}_{1-\mathrm{x}} \mathrm{Mg}_{\mathrm{x}} \mathrm{Te}$ single quantum well structures having a two-dimensional electron gas (2DEG) with mobility exceeding $10^{5} \mathrm{~cm}^{2} / \mathrm{Vs}$ [8]. These materials are also of interest for light emitting devices [9]. Degradation processes significantly deteriorate material quality and thus limit its industrial use for electronic devices. Our preliminary results have suggested that external hydrostatic pressure induces degradation of electrical properties of low-dimensional $\mathrm{CdTe} / \mathrm{Cd}_{1-\mathrm{x}} \mathrm{Mg}_{\mathrm{x}} \mathrm{Te}$ heterostructures grown on GaAs [10]. In contrast magneto-optical studies performed under hydrostatic pressure in the same range of pressures 
as in Ref. [10] and on similar heterostructures, i.e. $\mathrm{CdTe} / \mathrm{Cd}_{1-\mathrm{y}} \mathrm{Mn}_{\mathrm{y}} \mathrm{Te}$ grown on $\mathrm{GaAs}$, did not reveal any worsening of the optical properties of the samples [11].

The aim of the present work was to study the effect of external hydrostatic pressure on degradation of electrical and optical properties of low-dimensional $\mathrm{CdTe} / \mathrm{Cd}_{1-\mathrm{x}} \mathrm{Mg}_{\mathrm{x}} \mathrm{Te}$ heterostructures grown by molecular beam epitaxy (MBE) on GaAs substrates in order to understand the origin of this undesirable phenomenon. It can be expected that the very large lattice mismatch between a GaAs substrate and a CdTe layer, $\left(\mathrm{a}_{\mathrm{CdTe}}-\mathrm{a}_{\mathrm{GaAs}}\right) /<\mathrm{a}>=13,6 \%$ [12] will lead to formation of a large number of defects in the vicinity of the $\mathrm{CdTe} / \mathrm{GaAs}$ interface which results in a relaxation of CdTe-based epilayers. Application of external hydrostatic pressure can enhance the residual internal strains due to mismatch of the compressibility between GaAs and CdTe and thus accelerate the process of degradation, which occurs over time even without pressure. We believe that the origin of pressure induced degradation (different compressibilities) is similar to that observed due to cooling-heating temperature cycles (different thermal expansion coefficients) that also contributes to device ageing.

Our investigation procedure consisted of three steps. First, the samples were characterized at ambient pressure by means of magnetotransport measurements, photoluminescence (PL), X-ray diffraction and cross sectional transmission electron microscopy (TEM). Second, we applied external hydrostatic pressure while monitoring electrical properties of the samples. Third, after releasing the external pressure we repeated the characterization of the samples in order to find any persistent changes related to degradation. Experiments were performed on the same samples as in the first step except the TEM studies because in this case sample preparation is a destructive process. For TEM two samples from the same wafers were cut. One of them was studied after pressure application (step three), the second was not subjected to pressure (step one). 


\section{SAMPLES AND EXPERIMENT}

All the modulation doped structures were grown on (100) GaAs/CdTe hybrid substrates by molecular beam epitaxy using elemental sources of $\mathrm{Cd}(7 \mathrm{~N}), \operatorname{Te}(7 \mathrm{~N}), \operatorname{Mg}(5 \mathrm{~N})$ and a compound source of $\mathrm{ZnI}_{2}(5 \mathrm{~N})$ for iodine doping. The substrates were fabricated in separate MBE processes by covering the GaAs wafer first with $10 \AA$ of $\mathrm{ZnTe}$ and then by about $3 \mu \mathrm{m}$ of undoped CdTe (more details are given in Ref. [8]). Samples used in our studies are shown in Fig. 1. The $\mathrm{CdTe} / \mathrm{Cd}_{1-\mathrm{x}} \mathrm{Mg}_{\mathrm{x}} \mathrm{Te}$ structures consisted of a single $100 \AA$ - thick quantum well (QW) of CdTe embedded in $\mathrm{Cd}_{1-\mathrm{x}} \mathrm{Mg}_{\mathrm{x}} \mathrm{Te}$ barriers with magnesium composition of $x=0.13$. The quantum well was separated from the iodine - doped $\mathrm{Cd}_{1-\mathrm{x}} \mathrm{Mg}_{\mathrm{x}} \mathrm{Te}$ layer by an undoped spacer. The iodine donors supplying electrons were introduced into a $100 \AA$ - thick layer of the $\mathrm{Cd}_{1-\mathrm{x}} \mathrm{Mg}_{\mathrm{x}} \mathrm{Te}$ top barrier with a concentration of iodine donors of $5 \times 10^{17} \div 10^{18} \mathrm{~cm}^{-3}$. Parameters characterizing the samples are given in Table I. In the case of the \#sp800 sample we introduced three additional $100 \AA$ thick doped layers (with concentration $10^{18} \mathrm{~cm}^{-3}$ ) located deep in the $\mathrm{Cd}_{1-\mathrm{x}} \mathrm{Mg}_{\mathrm{x}} \mathrm{Te}$ bottom barrier, and separated from the quantum well by 1000 $\AA, 2000 \AA$ and $3000 \AA$, respectively [8].

For electrical measurements ohmic contacts were formed by annealing indium dots in air at $200-220{ }^{\circ} \mathrm{C}$ for about $30 \mathrm{~s}$. The shape of each sample was defined by photolithography. Measurements of the resistance tensor were performed in a magnetic field up to $7 \mathrm{~T}$ at temperature $4.2 \mathrm{~K}$. In order to apply external hydrostatic pressure the heterostructures were placed in a high pressure cell filled with a mixture of oil and kerosene as a pressure transmitting medium. Pressure (up to $1 \mathrm{GPa}$ ) was applied at room temperature. For performing magnetotransport measurements under pressure, the pressure cell was inserted into a cryostat and cooled down. After completing the measurements, the pressure cell was removed from the cryostat and heated up to room temperature. Then hydrostatic pressure was changed and the procedure was repeated. 


\section{RESULTS AND DISCUSSION}

\section{A. Sample characterization at ambient pressure.}

X-ray diffraction studies were performed using a high-resolution diffractometer [13] with the Bartels monochromator [14] using four 220 reflections from silicon. The theoretical angular resolution of such a monochromator for $\mathrm{CuK \alpha} \alpha$ radiation is equal to about 5 arcsec. The measurements showed that the full width at half maximum (FWHM) of the 004 rocking curves originating from the GaAs substrate was equal to about 8 arcsec, which was close to the theoretical value and thus evidenced high quality of the substrate material. The 004 rocking curves for the epilayer structure were much broader. The FWHM differed from sample to sample having values from 100 to about $230 \operatorname{arcsec}$ (see Fig.2).

Photoluminescence spectra were measured in the energy range: $1.38 \mathrm{eV}-1.92 \mathrm{eV}$ by means of a He-Cd laser and Oriel CCD matrix detector. Typical spectrum of a representative sample is presented in Fig. 4. The line at $1.65 \mathrm{eV}$ is related to optical transition in the quantum well while the lines at $1.74 \mathrm{eV}, 1.79 \mathrm{eV}$ and $1.82 \mathrm{eV}$ originate from the $\mathrm{Cd}_{1-\mathrm{x}} \mathrm{Mg}_{\mathrm{x}} \mathrm{Te}$ barrier and the buffer layer. Positions of the lines were somewhat sample dependent, however in each PL spectrum we could distinguish optical transitions in the QW and in the barrier.

The results of magnetotransport measurements are shown for representative heterostructures in Fig. 5. Two samples, \#m1 and \#m2 revealed well resolved quantum Shubnikov - de Haas ( $\mathrm{SdH})$ oscillations while for \#sp800 and \#I20 these oscillations were not observed. However, the non-oscillatory magnetic field dependence of the diagonal component of the conductance tensor, $\sigma_{\mathrm{xx}}$ was similar for all the samples - it decreased strongly at low magnetic field and then saturated at some constant value greater than zero. SdH oscillations and the observed strong magnetic field dependence of $\sigma_{\mathrm{xx}}$ at low fields are related to two-dimensional (2D) high mobility conduction in the quantum well, while the high field 
saturation of $\sigma_{\mathrm{xx}}$ proves the existence of some additional (parasitic) electron transport channel of much lower mobility in all the samples. A similar effect has been reported for $\mathrm{CdTe} / \mathrm{Cd}_{1-\mathrm{y}} \mathrm{Mn}_{\mathrm{y}} \mathrm{Te}$ quantum well structures [15] and for III-V heterostructures (e.g. [16] and references therein). The presence of these two conductance channels enabled us to monitor the process of degradation both in the quantum well and in the barrier. To analyze the contribution of 2D high mobility and parallel low mobility channels we assumed that both conductance channels are in intimate contact and therefore see the same electrical field [17]. In such a case the experimental data can be analyzed by means of the following formula:

$$
\sigma_{\mathrm{xx}}=\left(\mathrm{en}_{2 \mathrm{D}} \mu_{2 \mathrm{D}}\right) /\left(1+\left(\mu_{2 \mathrm{D}} \mathrm{B}\right)^{2}\right)+\sigma_{\|}
$$

where $\mathrm{n}_{2 \mathrm{D}}$ and $\mu_{2 \mathrm{D}}$ are two-dimensional sheet carrier density and mobility, respectively, $\mathrm{B}$ stands for magnetic field and $\sigma_{\|}$is the contribution of the conductance in the parallel channel taken as B-independent. The value of $\mathrm{n}_{2 \mathrm{D}}$ was extracted from the period of $\mathrm{SdH}$ oscillations, except the samples for which oscillations were not observed. In such cases $n_{2 D}$ together with $\mu_{2 \mathrm{D}}$ and $\sigma_{\|}$were fitted to get the best agreement between theoretical curves and experimental data. The fitting parameters are listed in Table I. The parameter $\sigma_{\|}$similarly to two-dimensional conductance, should be a function of magnetic field, the electron concentration, and the mobility. However, we found that in the available range of magnetic field it was almost field independent due to the very low value of the mobility. Therefore it appeared impossible to extract from our experimental data separately the values of the electron concentration and the mobility. We could only roughly estimate that the value of the mobility in the parallel conduction channel did not exceed $1000 \mathrm{~cm}^{2} /$ Vs. We found (see Table I) that the contribution of the conductance in the parallel channel increased as a function of spacer thickness. To explain the presence of the parallel electron transport channel we performed theoretical calculations of the conduction band energy profiles adopting numerical procedure used for InP/InGaAs heterostructures in Ref. [18]. Calculations have shown that for 
all our samples, like the case described in [18], a potential valley is formed spontaneously outside the QW in the region of doping with iodine donors. This leads to redistribution of free electrons between the quantum well and the valley and the appearance of an additional lowmobility conductance channel in the barrier.

\section{B. Magnetotransport study under pressure.}

The influence of external hydrostatic pressure on sample electrical properties was strong and similar for of all the investigated samples. In Fig. 6 we present the results for a representative heterostructure. For each curve two values of pressure are given, the first corresponds to pressure applied at room temperature, the second - to the pressure, after cooling the sample down to $4.2 \mathrm{~K}$. One can see a strong decrease of the conductance with pressure, which is accompanied (in the samples: $\# \mathrm{~m} 1$ and $\# \mathrm{~m} 2$ ) by a reduction of the amplitude of SdH oscillations. At sufficiently high pressures the oscillatory component of the conductance disappeared. We found that at low pressures, when the $\mathrm{SdH}$ oscillations were still visible, their period did not vary with pressure, which meant that carrier sheet density in the quantum well was almost pressure independent. The value of pressure at which the $\mathrm{SdH}$ oscillations disappeared differed slightly from sample to sample but was close to $0.7 \mathrm{GPa}$. Analysis of the experimental data by means of formula (1) showed that the main effect of the external pressure is a very strong decrease of the Hall mobility (as well as $\mu_{2 \mathrm{D}}$ mobility) from a value of the order of $10^{4} \mathrm{~cm}^{2} / \mathrm{Vs}$ down to about $20-50 \mathrm{~cm}^{2} / \mathrm{Vs}$ (shown for a representative sample in Fig. 7). This leads to significant lowering of the conductance in the quantum well. The value of the conductance in parallel channel, $\sigma_{\|}$, decreased with pressure by about one order of magnitude and we think that it was caused mainly by the decrease of the mobility. In the insert of Fig. 7. we compare the contribution of two types of conduction channels - the 2D high mobility channel, $\sigma_{2 \mathrm{D}}$ and the parallel low mobility one, $\sigma_{\|}$. The effect of pressure is 
more pronounced for $2 \mathrm{D}$ electron conduction in the quantum well. The conduction of the high mobility channel, $\sigma_{2 \mathrm{D}}$ falls strongly down and at high pressure only three-dimensional (3D) electron transport in the parallel channel is practically observed. This is contrary to the effect observed in III-V heterostructures [16].

\section{Properties of the samples after pressure treatment.}

All the changes described in chapter III.B persisted - after releasing external pressure the electrical properties of the samples did not return to their previous values but remained as measured at high pressure. The mobility (both Hall mobility and $\mu_{2 \mathrm{D}}$ ) after completing pressure experiments was very low (see open circle in Fig. 7), the conductance $\sigma_{\mathrm{xx}}$ achieved a very low value while the Hall resistance $\left(\rho_{\mathrm{xy}}\right)$ revealed a linear dependence on magnetic field (see Fig. 8). The magnetotransport results proved that external pressure induces formation of huge number of some electrically inactive defects in the whole $\mathrm{CdTe} / \mathrm{Cd}_{1-\mathrm{x}} \mathrm{Mg}_{\mathrm{x}} \mathrm{Te}$ heterostructure, which via the substantial reduction of the electron mobility, practically eliminates electron conduction in the quantum well. The X-ray diffraction studies indicated that some structural defects (e.g. dislocations or stacking faults), were created not only in the II-VI structure but also in GaAs substrate, which was evidenced as a pressure-induced increase of the GaAs rocking curve widths. For the substrate the FWHM of the rocking curve increased by about 5 arcsec reaching a value of 13 arcsec. In the case of the $C d T e / \mathrm{Cd}_{1-\mathrm{x}} \mathrm{Mg}_{\mathrm{x}} \mathrm{Te}$ heterostructure, pressure broadened the rocking curves by more than a factor of two for some samples (see Fig. 2).

TEM studies performed on a sample after pressure release showed a high density of stacking faults in the CdTe buffer layer. These stacking faults located on $\{111\}$ planes are clearly visible on a high resolution electron microscopy (HREM) image taken from the area of the GaAs/CdTe interface (Fig.3b). The highest density, of about $5 \pm 1 \times 10^{11} \mathrm{~cm}^{-2}$, of these 
stacking faults was observed in the area adjacent to this interface, their density gradually decreased with increasing distance from the interface, as can be seen in the low-magnification image taken from this sample (Fig.3c). For example, this density was estimated to be about $1.0 \pm 0.2 \times 10^{11} \mathrm{~cm}^{-2}$ at a distance of $200 \mathrm{~nm}$ away from the GaAs/CdTe interface. Despite gradual decrease of the density of stacking faults with increasing distance from the GaAs/CdTe interface, some faults were still observed even about $2 \mu \mathrm{m}$ from the interface.

A much lower density of such stacking faults located on $\{111\}$ planes was observed in a sample before pressure treatment as can be seen in Fig.3a. In fact, there is no single stacking fault visible in this image indicating that the density of these faults near the interface area is lower than $5.0 \pm 1.0 \times 10^{9} \mathrm{~cm}^{-2}$. One can expect that before pressure treatment such a low density of stacking faults was present not only in the interface area but also in the whole structure.

The photoluminescence experiments reveal a hydrostatic pressure induced decrease of the PL intensity by about $50 \% \pm 20 \%$ and a small energy shift of the PL lines without visible change of their width. The most pronounced shift by about $1.8 \pm 0.3 \mathrm{meV}$ towards lower energy was observed for the PL line related to optical transition in the QW. In the case of pressure induced formation of new defects it is difficult to analyze the intensity of the PL lines, however their displacement indicates that pressure changes internal strains. However, comparison of the photoluminescence experiments to electrical measurements shows that the effect of external pressure on optical properties is much weaker than on electrical ones and even application of the highest pressure, $\mathrm{p}=1 \mathrm{GPa}$ did not lead to disappearance of the photoluminescence or significant change of its shape.

Our results show unambiguously that high hydrostatic pressure induces degradation of $\mathrm{CdTe} / \mathrm{Cd}_{1-\mathrm{x}} \mathrm{Mg}_{\mathrm{x}} \mathrm{Te}$ heterostructures grown on GaAs substrates due to formation of a huge number of structural defects in the samples. Since the density of defects is largest in the 
vicinity of the II-VI/GaAs interface and increases by about two orders of magnitude after pressure experiments, we suggest that the degradation process originates from the mismatch of the compressibility between GaAs and CdTe. Pressure induced relative changes of the lattice constants are different for CdTe and GaAs, so that for $\mathrm{p}=1 \mathrm{GPa}(\Delta \mathrm{a} / \mathrm{a})_{\mathrm{CdTe}}-(\Delta \mathrm{a} / \mathrm{a})_{\mathrm{GaAs}}$ $=0.28 \%$. For comparison, an analogous value arising from the mismatch of linear thermal expansion coefficients for a temperature variation of $500 \mathrm{~K}$ is equal to about $0.10 \%$, i.e. almost 3 times smaller than that caused by pressure of $p=1 \mathrm{GPa}$ (lattice parameters are given in Table II). Both external hydrostatic pressure and temperature variations change the lattice constants of the GaAs substrate and the II-VI material by different amounts, which induces internal stresses and enhances existing residual stress in the vicinity of the GaAs/CdTe interface. These strains are relaxed by formation of structural defects like stacking faults and dislocations. Existing stacking faults and other defects near the interface, like the case of ZnSe grown on GaAs [1-7] are frequently the sources of new dislocations which propagate from the interface into the epitaxial epilayer [19] sometimes leading to internal micro-cracks. The micro-cracks and dislocations cut paths of conductance and cause increase of the resistance. In the case of quantum wells, due to their small thickness (100 $\AA)$ this effect leads to permanent elimination of electron conduction. There is however a crucial difference between electrical and optical effects. In the former case integrated values along the sample are measured. For example one micro-crack across the QW could completely switch off the electron transport and thus damage the sample from the point of view of its electrical properties. In the second case even many micro-cracks cannot influence significantly the optical properties.

Our results show that the mismatch of both compressibilities and thermal expansion coefficients (see Table I) between GaAs substrate and CdTe are the origin of degradation. In order to inhibit this process and thus to improve the II-VI material stability one has to use 
other substrates - e.g. substrates of CdTe-based II-VI compounds or GaAs compliant universal substrates (e.g. [20]).

\section{CONCLUSION}

We have shown that hydrostatic pressure leads to the formation of stacking faults, dislocations and other structural defects in the $\mathrm{CdTe} / \mathrm{Cd}_{1-\mathrm{x}} \mathrm{Mg}_{\mathrm{x}} \mathrm{Te}$ structure in the vicinity of the II-VI/GaAs interface due to the mismatch of the compressibility between a GaAs substrate and CdTe. These defects are dislocations, which propagate across the epilayer leading to internal micro-cracks and thus to permanent damage of the samples from the point of view of their electrical properties. In contrast - optical properties of the samples are much less influenced by this pressure-induced effect.

We believe that the degradation process of the $\mathrm{CdTe} / \mathrm{Cd}_{1-\mathrm{x}} \mathrm{Mg}_{\mathrm{x}} \mathrm{Te}$ structures over time due to cooling-heating temperature cycles is also related to the stresses induced near the substrate-heterostructure interface - in this case by the mismatch of thermal expansion coefficients. To avoid this undesirable phenomenon, we suggest use of substrates of a CdTebased compound or GaAs compliant universal substrates.

\section{ACKNOWLEDGEMENTS}

The authors gratefully acknowledge the assistance of Dr. W. Mac in the photoluminescence measurements and would like to thank Dr. T. Wojtowicz for useful discussions, Prof. J. Washburn for critical reading of the manuscript and L. Baj for preparation of masks for photolithography. This work was partially supported by the State Committee for Scientific Research (Republic of Poland) under Grant 2 P03B05215. One of us (J.J.) gratefully acknowledges the financial support of U.S. Department of Energy under the Contract No. DEAC03-76SF00098. 


\section{References}

[1] S. Guha, J.M. DePuydt, M.A. Haase, J. Qiu, and H. Cheng, Appl. Phys. Lett. 63, 3107 (1993).

[2] G.C. Hua, N. Otsuka, D.C. Grillo, Y. Fan, J. Han, M.D. Ringle, R.L. Gunshor, M. Hovinen, and A.V. Nurmikko, Appl. Phys. Lett. 65, 1331 (1994).

[3] H.T. Lin, D.H. Rich, and D.B. Wittry, J. Appl. Phys. 75, 8080 (1994).

[4] D. Seghier, J.S. Hauksson, H.P. Gislason, K.A. Prior, and B.C. Cavenett, J. Appl. Phys. 85, 3721 (1999).

[5] A.G. Kontos, E. Anastassakis, N. Chrysanthakopoulos, M. Calamiotou, and V.W. Pohl, J. Appl. Phys. 86, 412 (1999).

[6] D. Lüerßen, R. Bleher, H. Richter, Th. Schimmel, H. Kalt, A. Rosenauer, D. Litvinov, A. Kamilli, D. Gerthsen, K. Onkawa, B. Jobst, and D. Hommel, Appl. Phys. Lett. 75, 3944 (1999).

[7] M. Hovinen, J. Ding, A. Salokatve, A.V. Nurmikko, G.C. Hua, D.C. Li He, J. Han, M. Ringle, and R.L. Gunshor, J. Appl. Phys. 77, 4150 (1995).

[8] G. Karczewski, J. Jaroszyński, M. Kutrowski, A. Barcz, T. Wojtowicz, and J. Kossut, J. Cryst. Growth 184/185, 814 (1998).

[9] A. Waag, G. Bacher, A. Jakobs, A. Forchel, and G. Landwehr, J. Appl. Phys. 75, 5456 (1994).

[10] D. Wasik, J. Mikucki, J. Siwiec, M. Baj, J. Gronkowski, J. Jasiński, and G. Karczewski, High Pressure Research, Vol. 18, Number 1-6, page 95 (2000) Gordon \& Breach Science Publishers.

[11] H. Yokoi, S.W. Tozer, Y. Kim, D. Rickel, Y. Kakudate, S. Usuba, S. Fujiwara, S. Takeyama, G. Karczewski, T. Wojtowicz, and J. Kossut, J. Appl. Phys. 85, 5935 (1999). [12] Numerical Data and Functional Relationships in Science and Technology, Landolt- 
Börnstein, New Series Group III (Springer Verlag, Berlin, 1987) vol. 22 Pta.

[13] P.F. Fewster, J. Appl. Crystallogr. 22, 64 (1989).

[14] W.J. Bartels, J. Vac. Sci. Technol. B1, 338 (1983).

[15] S. Scholl, H. Schäfer, A. Waag, D. Hommel, K. von Schierstedt, B. Kuhn-Heinrich, and G. Landwehr, Appl. Phys. Lett. 62, 3010 (1993).

[16] D.K. Maude and J.C. Portal; High Pressure in Semiconductor Physics vol. II, ed. T. Suski \& W. Paul (Semiconductors and Semimetals, vol. 55, p.1 ed. Wilardson \& Beer).

[17] M. J. Kane, N. Apsley, D.A. Anderson, L.L. taylor and T. Kerr, J. Phys. C: Solid State Phys. 18, 5629 (1985).

[18] J. Mikucki, M. Baj, D. Wasik, W. Walukiewicz, W.G. Bi, and C.W. Tu, Phys. Rev. B61 7199 (2000).

[19] Y.H. Lo, Appl. Phys. Lett. 59, 2311 (1991).

[20] F.E. Ejeckam, M.L. Seaford, Y.-H. Lo, H.Q. Hou, and B.E. Hammons, Appl. Phys. Lett. 71, 776 (1997). 


\section{Table I.}

The samples used in the experiment. The meaning of the parameters is given in the text. The values of $n_{2 D}$ were determined from the period of $\mathrm{SdH}$ oscillations for the \#m1 and \#m2 samples, while for the rest samples from the fitting of the formula (1) to the experimental data.

\begin{tabular}{|c|c|c|c|c|c|c|c|}
\hline Sample & $\begin{array}{c}\text { cap layer } \\
\text { thickness }\end{array}$ & $\begin{array}{c}\text { Spacer } \\
\text { thickness }\end{array}$ & $\begin{array}{c}\mathbf{n}_{\mathbf{H}} \\
\left(\mathrm{cm}^{-2}\right)\end{array}$ & $\begin{array}{c}\mathbf{n}_{2 \mathrm{D}} \\
\left(\mathrm{cm}^{-2}\right)\end{array}$ & $\begin{array}{c}\boldsymbol{\mu}_{\mathrm{H}} \\
\left(\mathrm{cm}^{2} / \mathrm{Vs}\right)\end{array}$ & $\begin{array}{c}\boldsymbol{\mu}_{2 \mathrm{D}} \\
\left(\mathrm{cm}^{2} / \mathrm{Vs}\right)\end{array}$ & $\begin{array}{c}\boldsymbol{\sigma}_{\|} \\
(\square / \Omega)\end{array}$ \\
\hline$\# \mathrm{sp} 800$ & $1000 \AA$ & $800 \AA$ & $4.9 \times 10^{11}$ & $1.9 \times 10^{11}$ & 23200 & 36000 & $7.4 \times 10^{-4}$ \\
\hline$\# \mathrm{I} 20$ & $4500 \AA$ & $300 \AA$ & $1.4 \times 10^{11}$ & $0.9 \times 10^{11}$ & 15300 & 21800 & $1 \times 10^{-4}$ \\
\hline$\# \mathrm{~m} 1$ & $1000 \AA$ & $400 \AA$ & $4.4 \times 10^{11}$ & $2.4 \times 10^{11}$ & 31000 & 44800 & $1.9 \times 10^{-4}$ \\
\hline$\# \mathrm{~m} 2$ & $1500 \AA$ & $400 \AA$ & $4.8 \times 10^{11}$ & $3.6 \times 10^{11}$ & 10000 & 12300 & $2.5 \times 10^{-4}$ \\
\hline
\end{tabular}




\section{Table II.}

Lattice parameters of GaAs and CdTe taken from Ref. [12].

\begin{tabular}{|l|c|c|}
\hline & GaAs & CdTe \\
\hline Lattice constant, a & $5.653 \AA$ & $6.481 \AA$ \\
\hline Linear thermal expansion coefficient & $6.86 \cdot 10^{-6} 1 / \mathrm{K}$ & $4.75 \cdot 10^{-6} 1 / \mathrm{K}$ \\
\hline Bulk modulus & $75.4 \mathrm{GPa}$ & $46 \mathrm{GPa}$ \\
\hline
\end{tabular}




\section{Figure captions}

FIG. 1. Schema of the samples.

FIG. 2.. Rocking curves originating from the $\mathrm{CdTe} / \mathrm{Cd}_{1-\mathrm{x}} \mathrm{Mg}_{\mathrm{x}} \mathrm{Te}$ structure of the $\# \mathrm{~m} 1$ sample measured before (solid line) and after (dashed line) application of hydrostatic pressure of $\mathrm{p}=1$ GPa.

FIG. 3. TEM images of the GaAs/CdTe interface area of the \#m2 sample: (a) HREM image taken from as-grown sample and (b) HREM image together with (c) low-magnification, bright-field TEM image, both taken after pressure treatment.

FIG. 4. Photoluminescence spectra of the $\# \mathrm{ml}$ sample measured at $4.2 \mathrm{~K}$ and ambient pressure before (solid lines) and after (dash lines) application of hydrostatic pressure of $p=1$ GPa.

FIG. 5. Diagonal component of conductivity, $\sigma_{\mathrm{xx}}$ versus magnetic field measured on two representative $\mathrm{CdTe} / \mathrm{Cd}_{1-\mathrm{x}} \mathrm{Mg}_{\mathrm{x}} \mathrm{Te}$ samples at $4.2 \mathrm{~K}$ and ambient pressure. Fourier transform of the oscillations of sample \#m1 is shown in the insert.

FIG. 6. Diagonal component of conductivity, $\sigma_{\mathrm{xx}}$ of the $\# \mathrm{~m} 2$ sample measured at $4.2 \mathrm{~K}$ and various pressures. For each curve two values of pressure are given (see text). Curve denoted as APE was obtained at ambient pressure at $4.2 \mathrm{~K}$ after completing pressure experiments.

FIG. 7. Hall mobility of the \#m1 sample measured versus pressure at $4.2 \mathrm{~K}$ (solid circles). Open circle represents the Hall mobility measured at ambient pressure after completing pressure experiments. Contribution of low mobility parallel conductance, $\sigma_{\|}$- solid squares and two-dimensional one, $\sigma_{2 \mathrm{D}}$ - open squares are shown in the insert. Dotted lines are a guide to the eyes only.

FIG. 8. Hall resistance, $\rho_{x y}$ of two representative $\mathrm{CdTe} / \mathrm{Cd}_{1-\mathrm{x}} \mathrm{Mg}_{\mathrm{x}} \mathrm{Te}$ samples measured at 4.2 $\mathrm{K}$ and ambient pressure before (solid lines) and after (dashed lines) application of hydrostatic pressure of $\mathrm{p}=1 \mathrm{GPa}$. 
FIG. 1. Wasik, J. Appl. Phys.

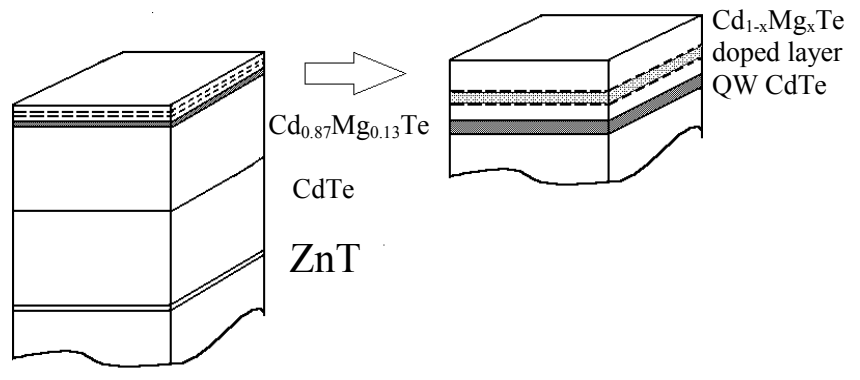


FIG. 2. Wasik, J. Appl. Phys.

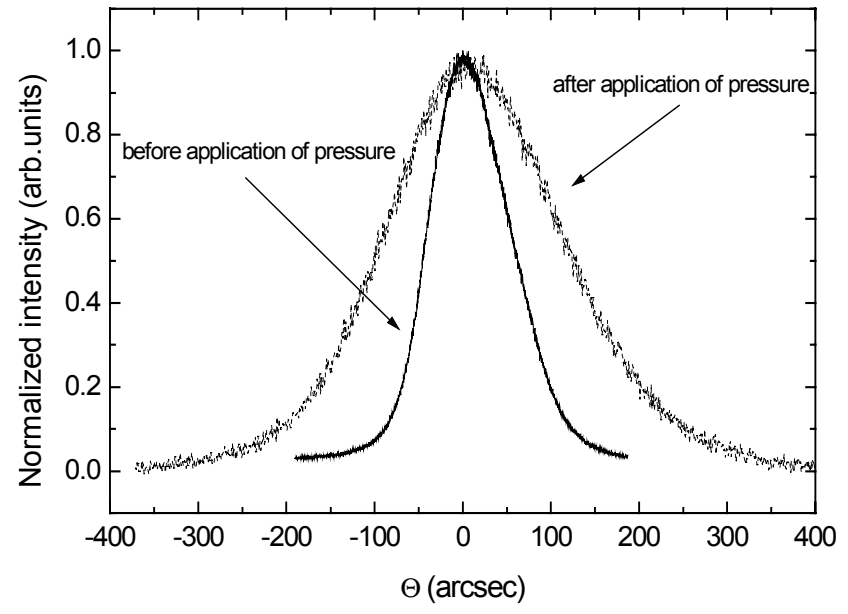


Fig. 3. Wasik, J. Appl. Phys.

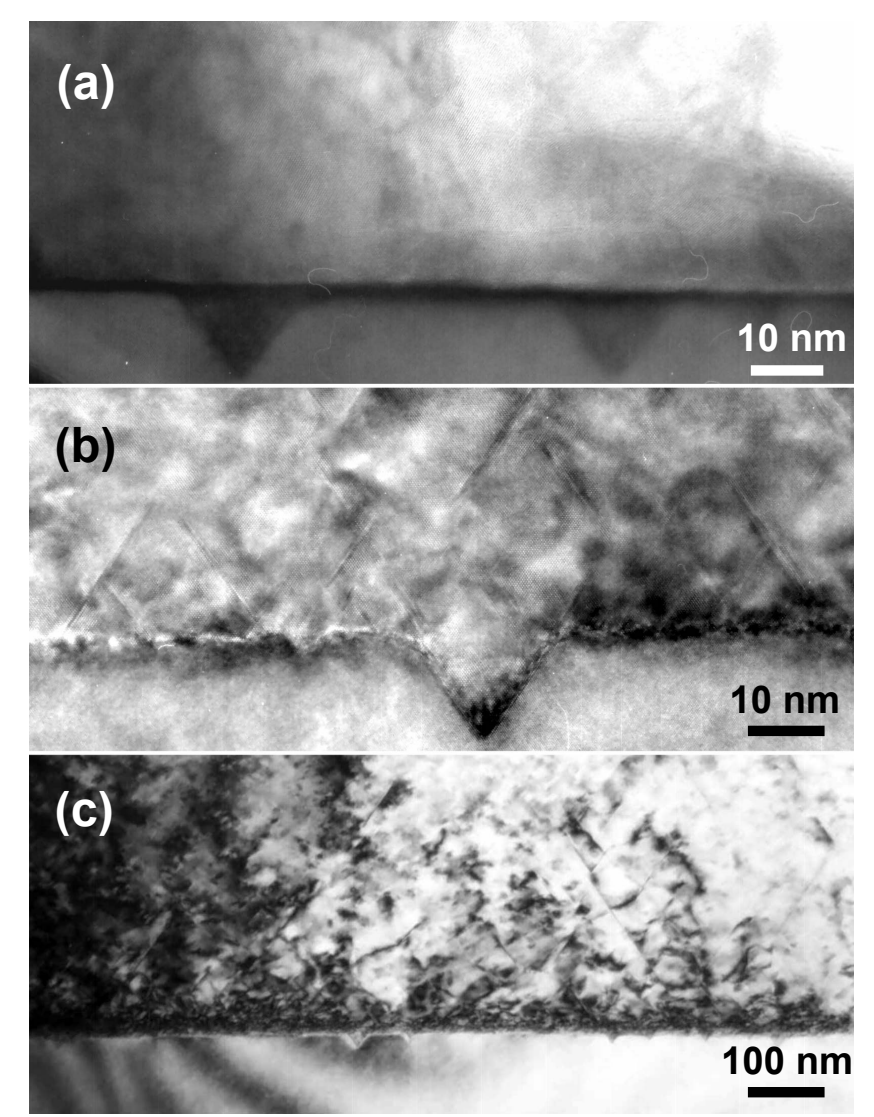


FIG. 4. Wasik, J. Appl. Phys.

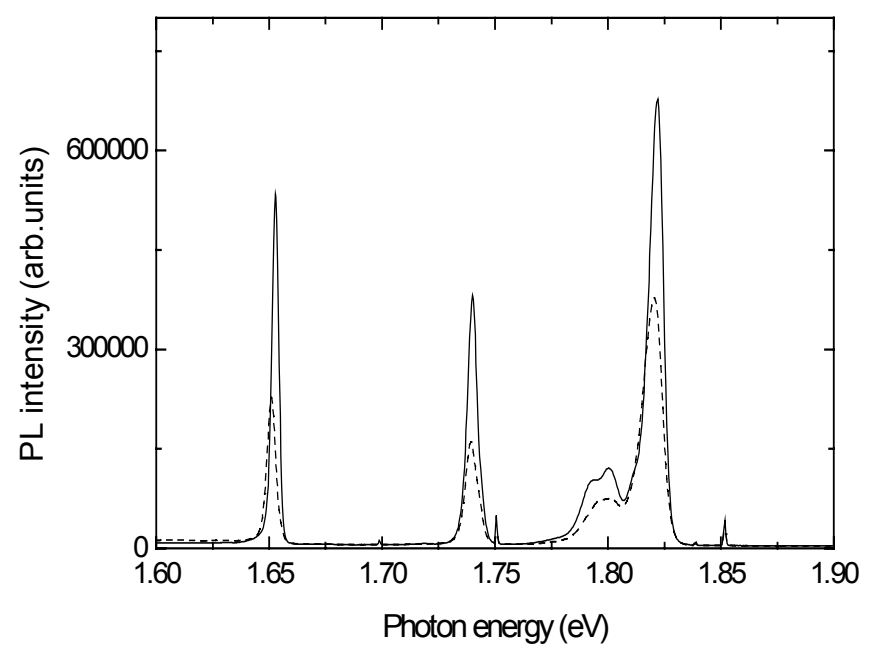


FIG. 5. Wasik, J. Appl. Phys.

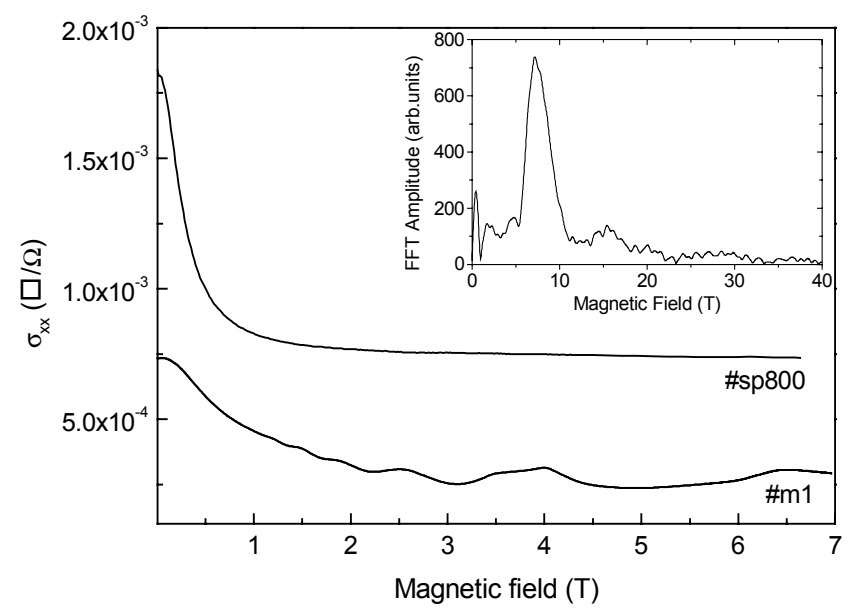


FIG. 6. Wasik, J. Appl. Phys.

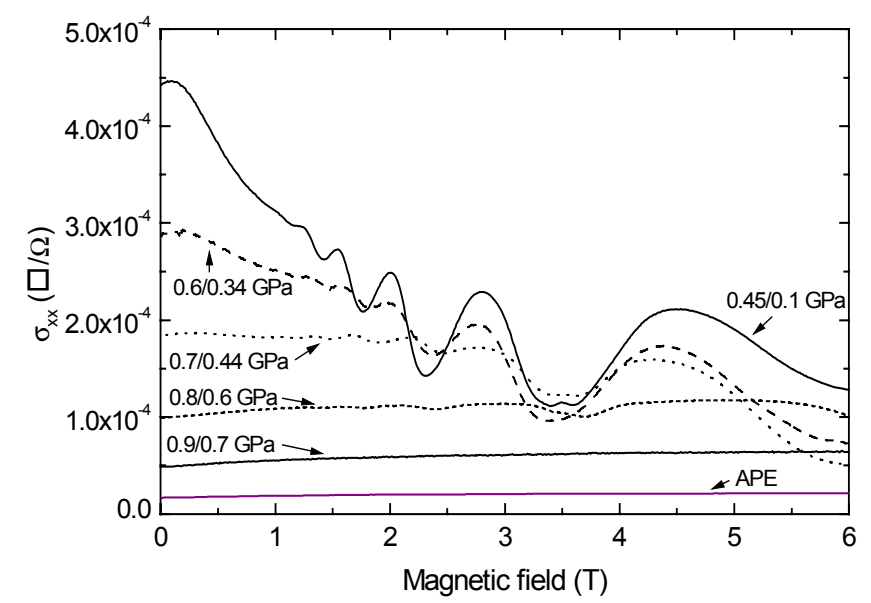


Fig. 7. Wasik, J. Appl. Phys.

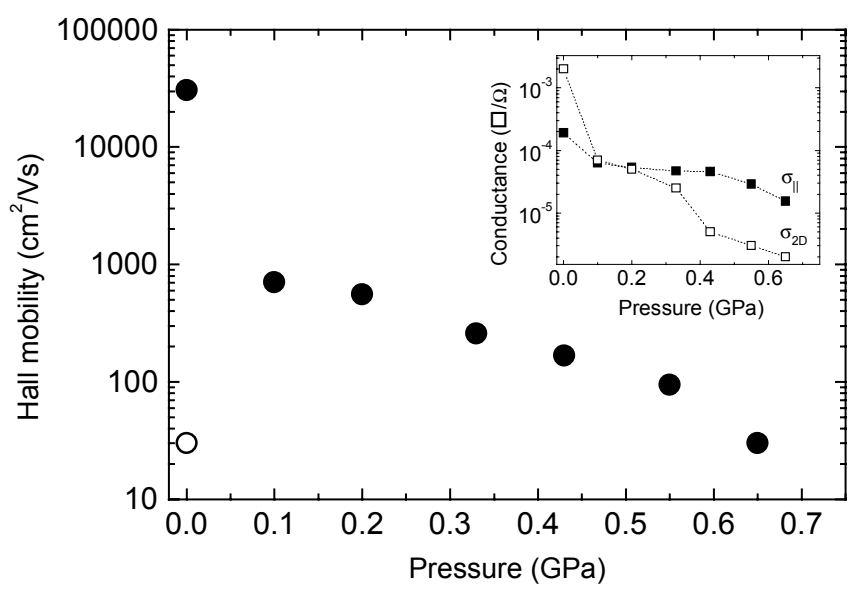


FIG. 8. Wasik, J. Appl. Phys.

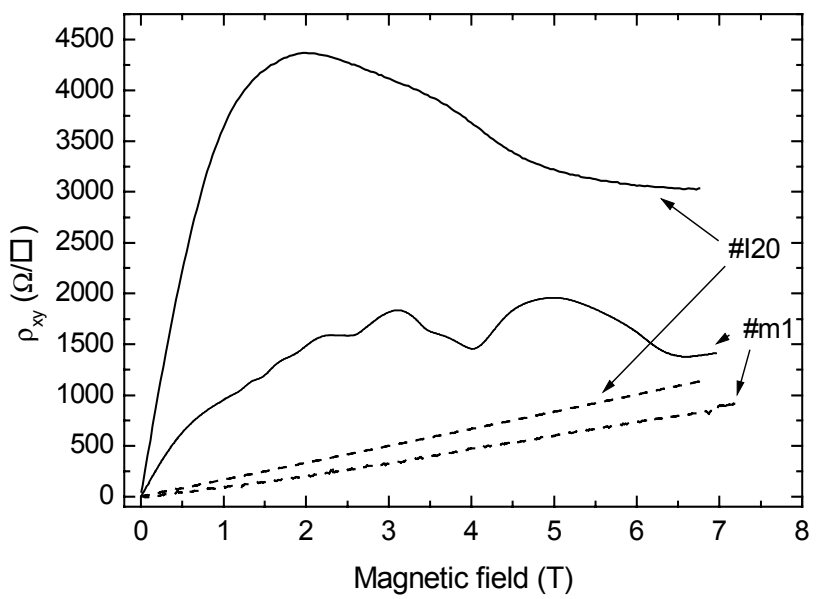

